\title{
Cell functional enviromics: Unravelling the function of environmental factors
}

\author{
Ana P Teixeira ${ }^{1,2 \dagger}{ }^{\dagger}$, João ML Dias ${ }^{3 \dagger}$, Nuno Carinhas ${ }^{1,2}$, Marcos Sousa $^{2}$, João J Clemente ${ }^{2}$, António E Cunha ${ }^{2}$, \\ Moritz von Stosch ${ }^{3}$, Paula M Alves ${ }^{1,2}$, Manuel JT Carrondo ${ }^{1,2}$ and Rui Oliveira ${ }^{1,3^{*}}$
}

\begin{abstract}
Background: While functional genomics, focused on gene functions and gene-gene interactions, has become a very active field of research in molecular biology, equivalent methodologies embracing the environment and geneenvironment interactions are relatively less developed. Understanding the function of environmental factors is, however, of paramount importance given the complex, interactive nature of environmental and genetic factors across multiple time scales.

Results: Here, we propose a systems biology framework, where the function of environmental factors is set at its core. We set forth a "reverse" functional analysis approach, whereby cellular functions are reconstructed from the analysis of dynamic envirome data. Our results show these data sets can be mapped to less than 20 core cellular functions in a typical mammalian cell culture, while explaining over $90 \%$ of flux data variance. A functional enviromics map can be created, which provides a template for manipulating the environmental factors to induce a desired phenotypic trait.
\end{abstract}

Conclusion: Our results support the feasibility of cellular function reconstruction guided by the analysis and manipulation of dynamic envirome data.

\section{Background}

The phenotype of a cell results from the combined effect of environmental and genetic factors [1,2]. While the investigation of the relationship between genetic and environmental factors at the molecular level has proved difficult [3], a number of recent systems biology studies have produced important insights about the nature of this complex relationship.

The relationship between genes and environment can be analysed by computational methods founded on the principle that the biochemical habitat of a cell is primarily "engraved" in its DNA sequence. Genes may be associated with metabolic enzymes, membrane transporters, signal transduction or regulatory control. Combined with basic biochemical information currently available in several databases (e.g. KEGG [4] and BioCyc databases [5]), it is possible to reconstruct the majority of the

\footnotetext{
* Correspondence: rui.oliveira@dq.fct.unl.pt

+ Contributed equally

'Instituto de Tecnologia Química e Biológica - Universidade Nova de Lisboa (ITQB-UNL), Av. República, Quinta do Marquês, 2781-901 Oeiras, Portugal Full list of author information is available at the end of the article
}

metabolic reaction network and also the associated exometabolome [6]. This is the currently accepted topdown, gene-to-function molecular biology paradigm.

Following these principles, Borenstein et al., [7] applied a graph-theoretical algorithm to determine these exogenously acquired compounds - they called it the seed set of an organism - for each of the 478 prokaryotic species with available metabolic networks in the KEGG database [4]. They found that about $8-11 \%$ of the compounds in the metabolic network of an organism correspond to the seed set and that each organism possesses a characteristic seed set. Moreover, comparing the seed set of the different organisms enabled them to trace the evolutionary history of both metabolic networks and growth environments across the tree of life, supporting the "reverse" ecology principle.

Using comparative genomics and flux balance analysis, Pal et al. [8] concluded that the adaptive evolution of bacterial metabolic networks in response to changing environments proceeds essentially by horizontal gene transfer (i.e. genes acquired from other species) of genes involved primarily in the transport and catalysis of
Ciomed Central

(C) 2011 Teixeira et al; licensee BioMed Central Ltd. This is an Open Access article distributed under the terms of the Creative Commons Attribution License (http://creativecommons.org/licenses/by/2.0), which permits unrestricted use, distribution, and reproduction in any medium, provided the original work is properly cited. 
external nutrients. With a similar approach, Kreimer et al. [9] studied the modularity of metabolic networks among 325 prokaryotic species with sequenced genomes. They observed that several environmental factors contributed to the variation in metabolic-network modularity across species. Their observations corroborated the findings of Pal et al. [8] that the variability in the natural habitat of an organism promotes modularity in its metabolic network.

Allen et al. [10] and Kell et al. [11] analysed the complete set of metabolites excreted or secreted by yeast cultures using high-throughput direct injection mass spectrometry. They called the technique "metabolic footprinting". They observed that a high number of metabolites, typically between 50-150 metabolites, are either excreted or secreted to the medium. Using statistical methods they showed that the information contained in the environment is sufficient to distinguish between different physiological states of wild type yeast strains and single-gene deletion mutants and concluded that the technique has high potential for functional analysis and metabolic engineering.

All the above studies concur with a profound, bidirectional link between environment and genes, with routes on the evolutionary principles of life. When exposed to a particular environment, a given genotype will respond and change its environment in a unique way, which can be distinguished even between singlegene deletion mutants [10]. Supported by these observations, we explored the feasibility of a bottom-up, envirome to cellular function reconstruction approach. Instead of using transcriptome data as in functional genomics, we set out to determine if it is possible to reconstruct cellular function from the analysis of envirome data sets alone. We term this reverse functional analysis approach "cell functional enviromics". In what follows, we describe the principles of the employed methodology and show how this approach can be applied to reconstruct Baby Hamster Kidney (BHK) metabolism.

\section{Results and Discussion}

A systems biology approach to cell functional enviromics In functional genomics, the goal is genome-wide cellular function reconstruction through the collection and analysis of transcriptome or proteome data over time. In functional enviromics, we attempt to bridge environmental factors and function through the collection and analysis of dynamic envirome data. The necessary elements to such an analysis are: i) setting the universe of cellular functions and envirome components, ii) collecting informative envirome data over time, and iii) systems level analysis of dynamic envirome data to find relationships between environmental variables and cellular functions.
To support such a framework, we have developed a computational algorithm called "envirome-guided projection to latent pathways (PLP)". "Cellular function" has been defined in different ways, here we adopted elementary flux modes (EM) as function descriptors [12-18] because they enable systematic, large-scale computational analysis of biochemical networks from a functional viewpoint. Mathematically, EMs form the convex basis of the null space solution of a metabolic network stoichiometric matrix. Biologically, they represent elementary network topologies defining all possible independent operational modes of a cell. EMs have been previously used to support computational functional genomics $[19,20]$. Here we apply the same rational to derive a functional enviromics algorithm.

A medium scale network has typically a very large number of EMs in the order of $\sim 10^{6}$ [21]. It is however unlikely that all of these cellular functions are active at the same time. In reality, despite the apparent complexity of cellular networks, only a very limited number of core network topologies are capable of robustly executing any particular biological function [22]. It is thus rational to assume that a given environmental stimulus activates only a moderate number of characteristic EMs.

In conformity with the above enumerated principles, PLP was designed to maximise the covariance between environmental state (envirome data sets) and observed phenotypic trait (rate of change of envirome variables) under the constraint of known genes translated into a plausible set of cellular functions (Figure 1). Simply put, PLP implements a discrimination algorithm to find a minimal set of EMs based on two criteria: i) variance of measured phenotypic trait explained by each EM and ii) degree of correlation of each EM with the environmental state. By maximising these two criteria, the algorithm delivers a ranking of EMs in increasing order of percentage of explained variance in the measured flux data, and a functional enviromics map (FEM) representing the strength of up- or down-regulation of EMs by environmental factors. Mathematical details of the method can be found in the Methods section. In what follows we illustrate the methodology with an application to a recombinant $\mathrm{BHK}$ cell line expressing the fusion glycoprotein IgG1-IL2 (see Methods for details).

\section{Envirome guided metabolic reconstruction of BHK cells}

The methodology was first applied to the analysis of a data set comprising measurements of 27 environmental factors collected from six independent, differently operated culture experiments as described in Methods and Table 1 . The main objective was to identify the "active" set of EMs by iteratively projecting the metabolism into a minimal set of core cellular functions that correlate with the envirome. The predictor (input) matrix included an 


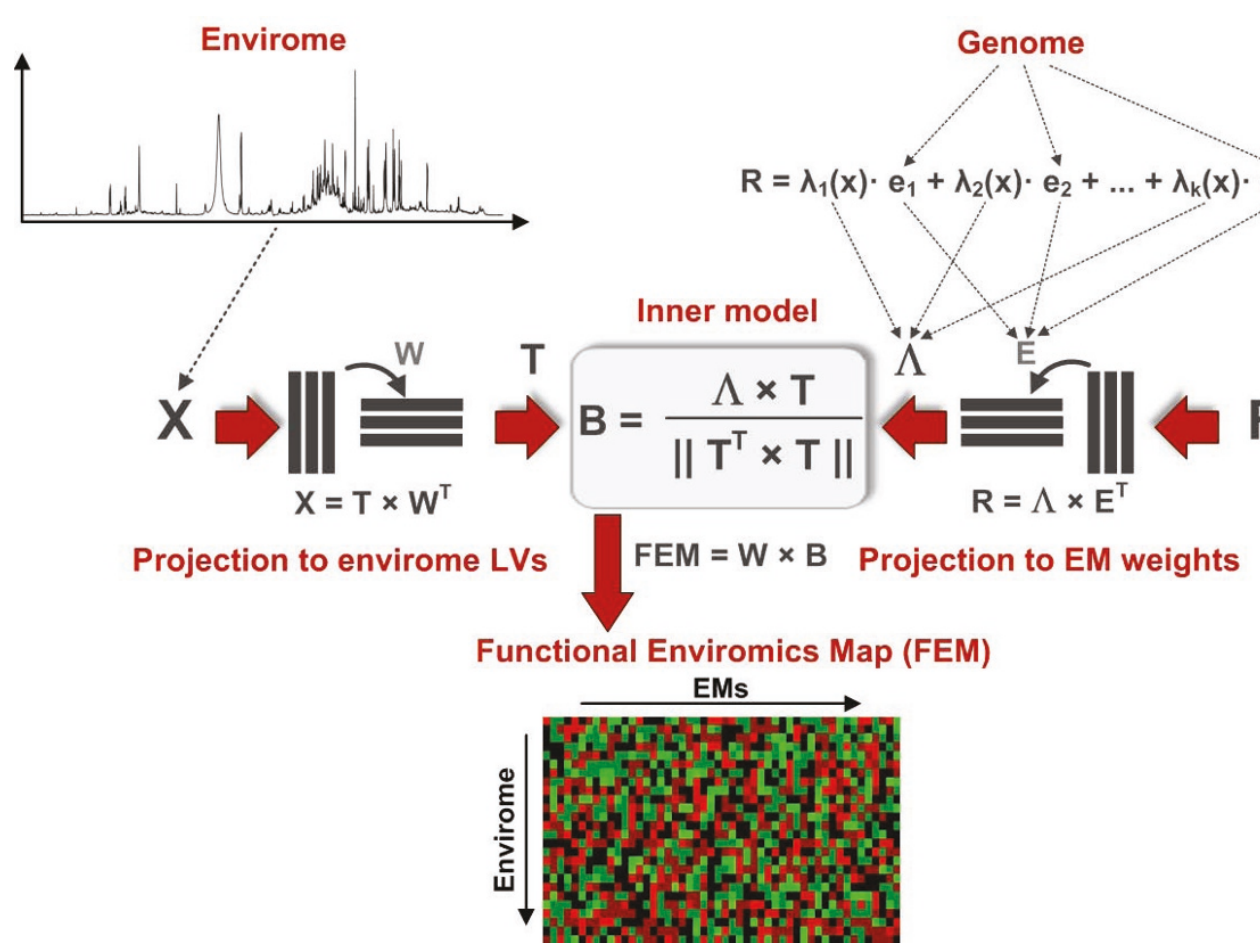

Figure 1 Schematic representation of a cell functional enviromics algorithm. The aim is to maximise the covariance between measured environmental factors $(X)$ and rate of change of environmental factors $(R)$ under the constraint of known genes translated into a plausible set of elementary flux modes (E). As in conventional partial least squares, the input matrix $(X)$ is decomposed into a loadings matrix of latent variables (W) and a scores matrix (T). The response data (R), however, are decomposed into genome dependent factors (the structure of elementary flux modes, E) and envirome dependent factors (weights of elementary flux modes, $\Lambda$ ). Then, only the envirome dependent factors $(\Lambda)$ are linearly regressed against envirome data $(X)$. Finally, such regression coefficients are organized into a functional enviromics map (FEM) with columns representing EMs, rows environmental factors and each element representing the strength of up- or down-regulation of each core cellular function by each envirome factor. Mathematical details can be found as Methods.

Table 1 Operating conditions of the fed-batch experiments

\begin{tabular}{|c|c|c|c|c|c|c|}
\hline & & $\begin{array}{c}\text { Fed- } \\
\text { batch } 1\end{array}$ & $\begin{array}{c}\text { Fed- } \\
\text { batch } 2\end{array}$ & Fed-batch 3 & $\begin{array}{c}\text { Fed- } \\
\text { batch } 4\end{array}$ & $\begin{array}{c}\text { Fed- } \\
\text { batch } 5\end{array}$ \\
\hline \multirow{6}{*}{$\begin{array}{l}\text { Initial concentration } \\
(\mathrm{mM})\end{array}$} & $\mathrm{Glc}_{\mathrm{i}}$ & 5.5 & 5.7 & 0.18 & 6.2 & 4.8 \\
\hline & $\mathrm{Gln}_{\mathrm{i}}$ & 0.2 & 0.5 & 0.42 & 3.9 & 0.1 \\
\hline & $\mathrm{Glu}_{\mathrm{i}}$ & 1.6 & 0.6 & 0.82 & 0.6 & 4.0 \\
\hline & $\operatorname{Ser}_{\mathbf{i}}$ & 2.3 & 1.3 & 1.30 & 1.4 & 1.4 \\
\hline & $\mathrm{Asp}_{\mathrm{i}}$ & 1.4 & 0.8 & 0.65 & 0.8 & 0.8 \\
\hline & $\begin{array}{c}\text { First feed } \\
\text { Started when Glc and/or Gln went below } \\
0.15 \mathrm{mM}\end{array}$ & $\begin{array}{l}\text { Glc }= \\
183.0 \\
\text { Gln }= \\
15.0 \\
\text { Glu }= \\
20.0\end{array}$ & $\begin{array}{c}\mathrm{Glc}= \\
182.0 \\
\mathrm{Gln}=5.2 \\
\mathrm{Glu}= \\
31.0\end{array}$ & $\begin{array}{c}\mathrm{Glc}=150.0 \\
\mathrm{Gln}=4.2 \\
\mathrm{Glu}=29.2\end{array}$ & $\begin{array}{l}\mathrm{Glc}= \\
198.0\end{array}$ & $\begin{array}{l}\mathrm{Glc}= \\
183.0\end{array}$ \\
\hline $\begin{array}{l}\text { Composition of feeds } \\
\text { (mM) }\end{array}$ & $\begin{array}{c}\text { Second feed } \\
\text { Started when Ser became limiting }(\sim 75 \mathrm{~h} \\
\text { of operation) }\end{array}$ & - & $\begin{array}{c}\mathrm{Glc}= \\
193.0 \\
\text { Gln }=4.5 \\
\text { Glu }= \\
36.0 \\
\text { Ser }=33.0 \\
\text { Cys }=8.5\end{array}$ & $\begin{array}{c}\mathrm{Glc}=160.0 \\
\mathrm{Gln}=5.4 \\
\mathrm{Glu}=25.6 \\
\text { Culture medium } 5 \text { times } \\
\text { concentrated }\end{array}$ & $\begin{array}{l}\text { Glc }= \\
156.0 \\
\text { Gln }= \\
42.8\end{array}$ & - \\
\hline
\end{tabular}


a

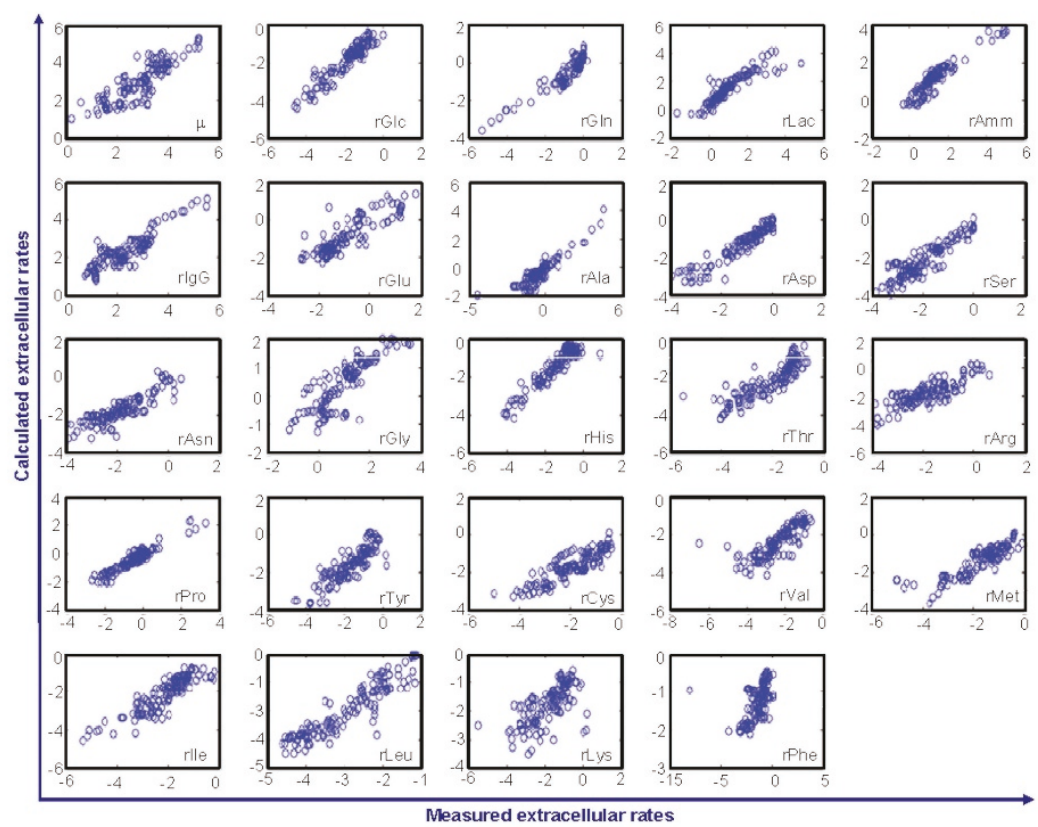

b

\begin{tabular}{|rc|}
\hline EM & $\begin{array}{c}\text { Added } \\
\text { variance }(\%)\end{array}$ \\
\hline 20 & 64.1 \\
1 & 70.5 \\
11 & 75.6 \\
6 & 79.2 \\
220 & 81.3 \\
4 & 83.0 \\
82 & 84.3 \\
7 & 85.3 \\
210 & 86.3 \\
12 & 87.4 \\
70 & 88.2 \\
5 & 88.9 \\
9 & 89.6 \\
43 & 90.2 \\
2 & 90.8 \\
117 & 91.3 \\
113 & 91.7 \\
3 & 92.2 \\
74 & 92.6 \\
79 & 93.0 \\
\hline
\end{tabular}

Figure 2 Application of the PLP algorithm to the analysis of envirome data collected from six BHK cultures as described in Methods and Table 1. Data from the growth stage of every experiment were pooled together. (a) Correlation between measured extracellular rates (R) and predicted rates obtained by reconstruction of fluxes of discriminated EMs. Units are in nmol.(10 $10^{6} \mathrm{cells}$.h $)^{-1}$. (b) Ranking of first 20 EMs that correlate with the envirome, explaining $93 \%$ of the variance in the measured extracellular rates. These pathways correspond to typical routes known to be active in cultured mammalian cell lines. Refer to the text for a description.

Table 2 Ranking of statistically significant EMs for sequential metabolic phases of fed-batch 3

\begin{tabular}{|c|c|c|c|c|c|c|c|c|c|}
\hline \multicolumn{2}{|c|}{ Phase I } & \multicolumn{2}{|c|}{ Phase II } & \multicolumn{2}{|c|}{ Phase III } & \multicolumn{2}{|c|}{ Phase IV } & \multicolumn{2}{|c|}{ Phase V } \\
\hline$\overline{E M}$ & $\begin{array}{l}\text { Var } \\
\text { (\%) }\end{array}$ & EM & $\begin{array}{l}\text { Var } \\
\text { (\%) }\end{array}$ & EM & $\begin{array}{l}\text { Var } \\
\text { (\%) }\end{array}$ & EM & $\begin{array}{l}\text { Var } \\
\text { (\%) }\end{array}$ & EM & $\begin{array}{l}\text { Var } \\
\text { (\%) }\end{array}$ \\
\hline 110 & 61.76 & 250 & 65.02 & 62 & 47.28 & 62 & 80.69 & 62 & 84.30 \\
\hline 6 & 69.92 & 1 & 89.68 & 1 & 90.61 & 12 & 85.98 & 6 & 89.43 \\
\hline 11 & 75.82 & 41 & 93.81 & 11 & 92.80 & 1 & 89.37 & 1 & 94.44 \\
\hline-7 & 81.63 & 28 & 96.57 & 6 & 93.96 & 86 & 91.08 & 49 & 97.04 \\
\hline 2 & 87.34 & 43 & 97.04 & 80 & 95.61 & 5 & 92.29 & 201 & 97.53 \\
\hline 5 & 91.93 & -4 & 97.40 & 5 & 96.28 & 43 & 93.11 & 119 & 97.84 \\
\hline 4 & 94.05 & 5 & 97.63 & 43 & 96.86 & 7 & 93.48 & -4 & 98.14 \\
\hline 101 & 95.86 & 47 & 97.84 & 7 & 97.07 & 6 & & 11 & 98.43 \\
\hline 43 & 96.45 & 6 & 98.01 & 201 & 97.14 & 69 & 94.00 & 43 & 98.67 \\
\hline 1 & 97.02 & 11 & 98.13 & -4 & 97.22 & 11 & 94.12 & 102 & 98.87 \\
\hline 86 & 97.55 & 80 & 98.24 & 3 & 97.27 & 225 & 94.16 & 5 & 98.95 \\
\hline 45 & 97.67 & 102 & 98.29 & 45 & 97.31 & -4 & 94.19 & 7 & 99.03 \\
\hline 33 & 97.77 & 15 & 98.31 & 102 & 97.34 & 102 & 94.27 & 87 & 99.11 \\
\hline 24 & 97.83 & 7 & 97.34 & 86 & 97.35 & 79 & 94.30 & 15 & 99.15 \\
\hline 41 & 97.89 & 87 & 97.35 & 81 & 97.36 & & & 37 & 99.23 \\
\hline 74 & 97.91 & 10 & 97.36 & 88 & 97.37 & & & 14 & 99.27 \\
\hline 3 & 97.92 & 9 & 97.37 & & & & & & \\
\hline
\end{tabular}

The minus sign indicates flux in the opposite direction for those EMs whose participating reactions are all reversible. EMs in bold were conserved between cultures and also selected from the full data set (see Figure $2 \mathrm{~b}$ ), showing the ability of the PLP algorithm to identify essential common features in metabolism. extensive list of environmental factors including: temperature, $\mathrm{pH}$, osmolality and concentrations of 24 measured components (viable cells, glucose, lactate, ammonia, glycoprotein and 19 amino acids). The target (output) matrix consisted of the 24 exchange fluxes calculated from the profiling of extracellular concentrations. The results are shown in Figure 2. The explained flux variance was calculated according to Eq. (4). It represents the variance in the flux data that is explained by a model built on the selected EMs.

A high degree of correlation was observed between measured envirome components and relative weight of discriminated EMs, ultimately reflected in high correlation between measured and predicted extracellular rates (Figure 2a). Within the universe of 251 possible EMs, 20 of them captured up to $93 \%$ of the measured rates variance (Figure $2 \mathrm{~b}$ ), even though the data were pooled together from several independent cultivation experiments, with inherently diverse environmental trajectories. The first selected EM is one of many for biomass synthesis (see Tables 2 and 3), describing more than $64 \%$ of total rate data variance. This concurs with the fact that most of the processed carbon is involved in biomass synthesis. The single EM for product formation (EM 1) is also selected, explaining part of the specific consumption rates of every amino acid. The EM 


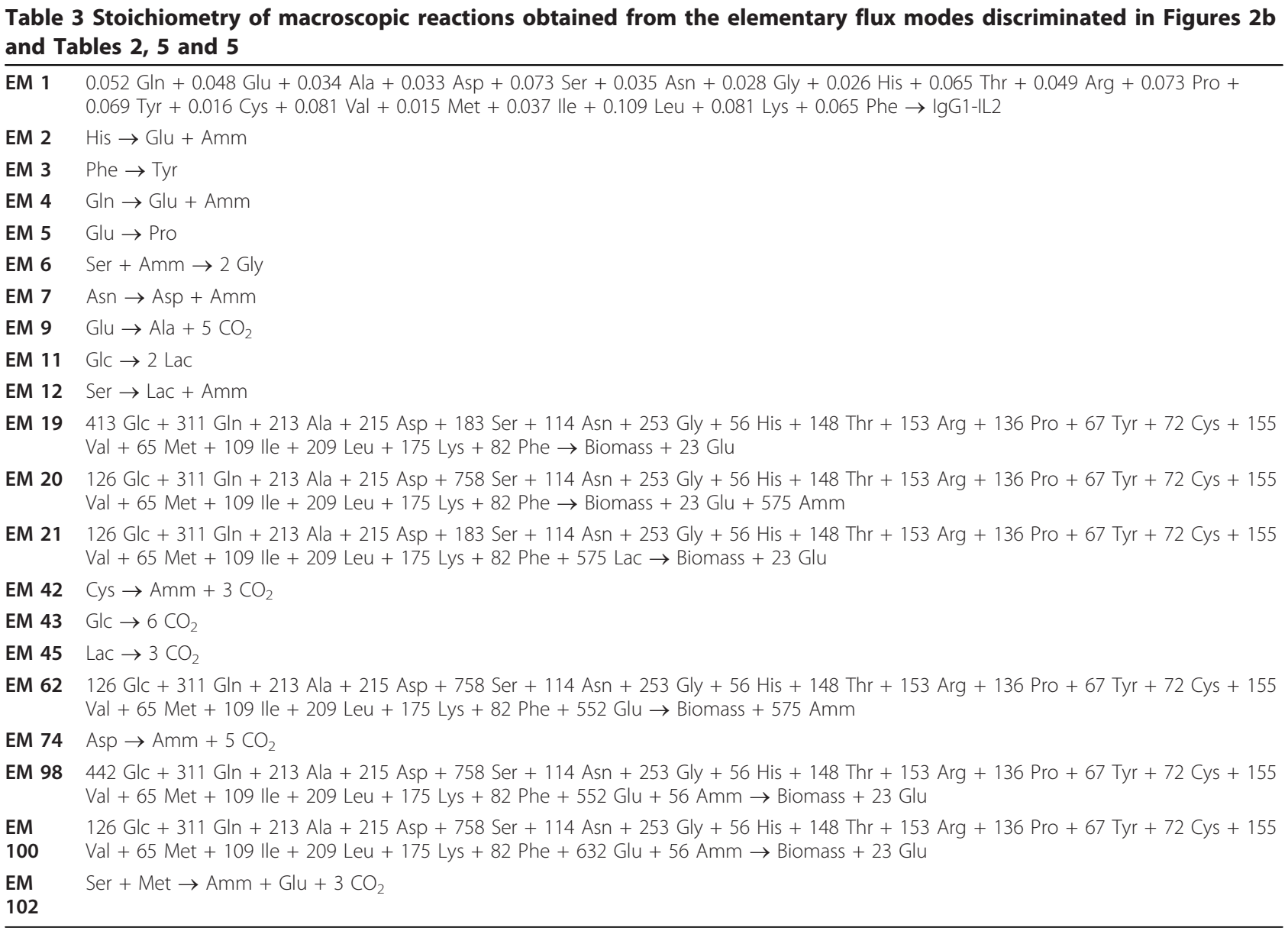

The stoichiometry of EM macroscopic reactions considers only extracellular compounds, and is readily calculated as described in Methods. Some of these pathways involve only a single intracellular reaction able to operate in steady-state, whenever all the metabolites involved are also present extracellularly.

corresponding to anaerobic conversion of glucose into lactate was selected in third place (EM 11). Given the low saturation constants of glucose transporters $(\mathrm{Km}=$ 1-2 mM) $[23,24])$, this pathway usually carries a high flux in mammalian cell cultures, leading to waste lactate formation. Serine transamination into glycine was the fourth selection (EM 6). Indeed, the rates of serine consumption and glycine production were quite high, mainly during the initial periods of culture. This pathway may represent a strategy for ammonia detoxification, the main toxic by-product in mammalian cell culture. The sixth position (EM 4) corresponds to the pathway of glutaminolysis, well known as a major carbon source for energy production in mammalian cells $[25,26]$. Complete oxidation of glucose in the TCA cycle (EM 43) is also selected, reflecting the metabolic shift induced during glucose fed-batch experiments.

Several other methods were proposed to calculate EMs weighting factors from flux vectors but none of these methods use the correlation with the envirome as criterion for EMs discrimination. As pointed out by Wiback et al. [27], the extreme pathways (and elementary modes) weighting factors used to reconstruct a particular flux vector may not be unique. Linear programming methods can be applied to calculate the minimum and maximum values of such weighting factors, thus defining a range of possible values for each pathway, called the $\alpha$-spectrum [27]. Unique solutions can also be obtained if additional assumptions about the biological system are considered. For instance, Nookaew et al. [28] proposed the determination of weighting factors by mixed integer linear programing to maximise the number of elementary modes that explain measured yield values. Using a different strategy, Schwartz and Kanehisa [29] proposed a quadratic program to minimize the sum of squares of weighting factors. To better illustrate our method, we compared it with the method proposed by Schwartz and Kanehisa [29], which basically selects the elementary modes that are closest to the actual biological sate by minimising the respective weighting factors. The application of this method to the 134 flux vectors data points identifies consistent subsets of 105 and 57 EMs with weighting factors bigger than 0.1 and 1.0 respectively (Figure 3a). As expected, this 


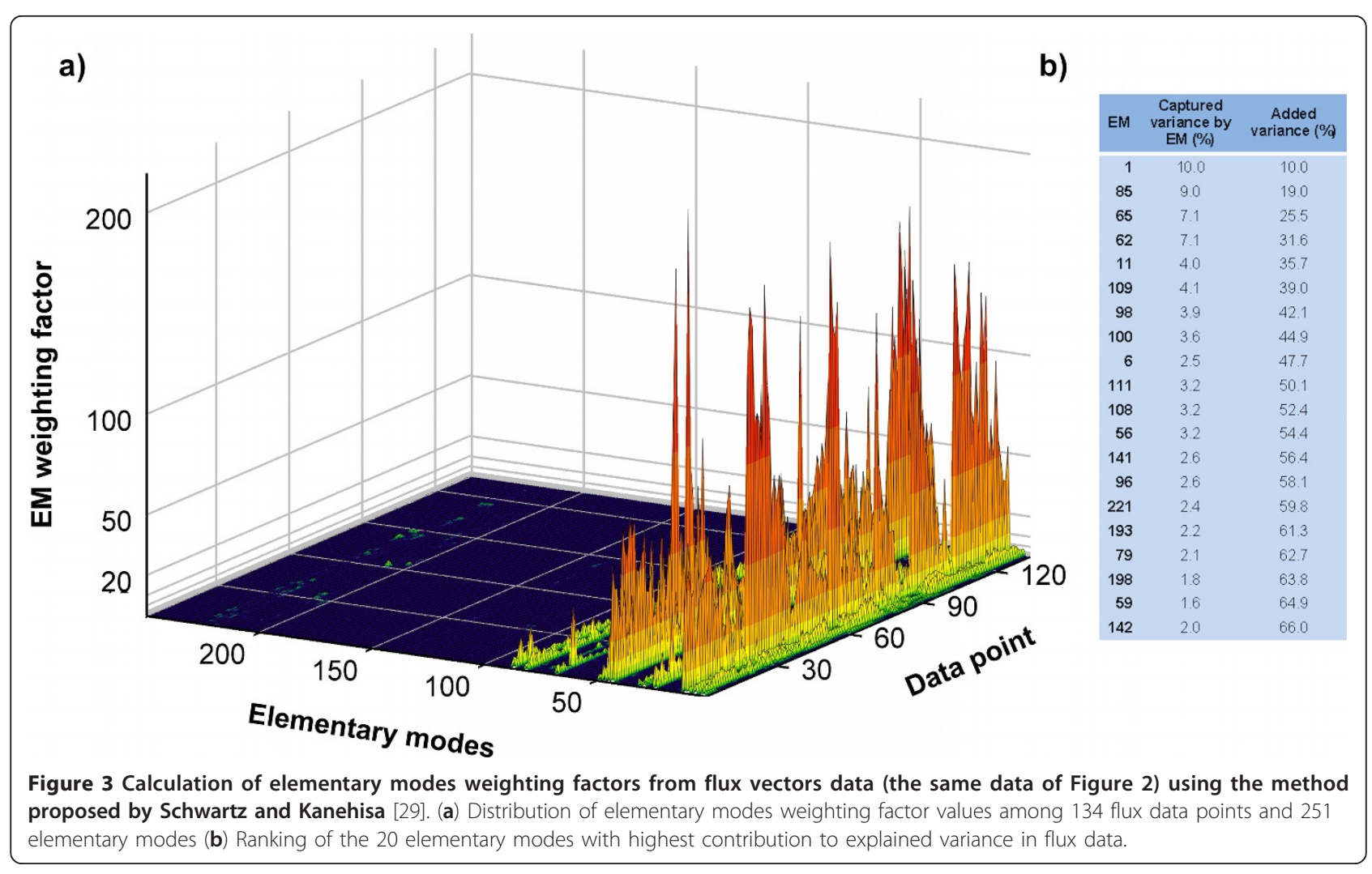

Table 4 Selection of active EMs during the growth for Fed-batch 1

\begin{tabular}{cccccccc}
\hline \multicolumn{9}{c}{ Fed-Batch 1 } \\
\hline \multicolumn{2}{c}{ Phase I } & \multicolumn{2}{c}{ Phase II } & \multicolumn{2}{c}{ Phase III } & \multicolumn{2}{c}{ Phase IV } \\
\hline EM & Var (\%) & EM & Var (\%) & EM & Var (\%) & EM & Var (\%) \\
\hline $\mathbf{2 0}$ & 80.69 & $\mathbf{5 3}$ & 58.03 & $\mathbf{6 2}$ & 69.34 & $\mathbf{6 2}$ & 94.84 \\
$\mathbf{2}$ & 84.24 & $\mathbf{1}$ & 92.43 & $\mathbf{1}$ & 82.00 & $\mathbf{1}$ & 96.22 \\
$\mathbf{- 7}$ & 87.54 & $\mathbf{6}$ & 93.65 & $\mathbf{6}$ & 88.68 & $\mathbf{6}$ & 97.51 \\
$\mathbf{8 6}$ & 90.79 & $\mathbf{7 4}$ & 94.76 & $\mathbf{1 7 1}$ & 92.55 & $\mathbf{4 9}$ & 98.49 \\
$\mathbf{2 0 7}$ & 91.81 & $\mathbf{1 1}$ & 95.54 & $\mathbf{2 4}$ & 94.38 & $\mathbf{4 3}$ & 99.21 \\
$\mathbf{6}$ & 92.79 & $\mathbf{- 4}$ & 96.25 & $\mathbf{4 3}$ & 96.05 & $\mathbf{2 4}$ & 99.36 \\
$\mathbf{1}$ & 93.48 & $\mathbf{2 6}$ & 96.95 & $\mathbf{7}$ & 96.70 & $\mathbf{2 6}$ & 99.42 \\
$\mathbf{1 1}$ & 93.88 & $\mathbf{1 0 2}$ & 97.43 & $\mathbf{- 4}$ & 97.01 & $\mathbf{2 2 1}$ & 99.46 \\
$\mathbf{- 5}$ & 94.00 & $\mathbf{2}$ & 97.84 & $\mathbf{1 0 2}$ & 97.29 & $\mathbf{7}$ & 99.50 \\
$\mathbf{1 0 2}$ & 94.14 & $\mathbf{2 2 0}$ & 98.08 & $\mathbf{5}$ & 97.52 & $\mathbf{8 7}$ & 99.54 \\
$\mathbf{3}$ & 94.22 & $\mathbf{4 1}$ & 98.25 & $\mathbf{2}$ & 97.72 & $\mathbf{1 1}$ & 99.56 \\
$\mathbf{9}$ & 94.27 & $\mathbf{7}$ & 98.39 & $\mathbf{1 1}$ & 97.91 & $\mathbf{4}$ & 99.57 \\
$\mathbf{- 4}$ & 94.31 & $\mathbf{4 5}$ & 98.45 & & & $\mathbf{5}$ & 99.57 \\
$\mathbf{7 2}$ & 94.34 & $\mathbf{4 3}$ & 98.50 & & & $\mathbf{2}$ & 99.58 \\
& & $\mathbf{- 5}$ & 98.52 & & & & \\
& & $\mathbf{3}$ & 98.55 & & & & \\
\hline
\end{tabular}

See Table 7 for time intervals.
Table 5 Selection of active EMs during the growth for Fed-batch 2

\begin{tabular}{|c|c|c|c|c|c|c|c|c|c|}
\hline \multicolumn{10}{|c|}{ Fed-Batch 2} \\
\hline \multicolumn{2}{|c|}{ Phase I } & \multicolumn{2}{|c|}{ Phase II } & \multicolumn{2}{|c|}{ Phase III } & \multicolumn{2}{|c|}{ Phase IV } & \multicolumn{2}{|c|}{ Phase V } \\
\hline EM & $\begin{array}{l}\text { Var } \\
(\%) \\
\end{array}$ & EM & $\begin{array}{l}\text { Var } \\
(\%) \\
\end{array}$ & EM & $\begin{array}{l}\text { Var } \\
(\%) \\
\end{array}$ & EM & $\begin{array}{l}\text { Var } \\
(\%) \\
\end{array}$ & EM & $\begin{array}{l}\text { Var } \\
(\%)\end{array}$ \\
\hline 45 & 61.90 & 62 & 92.01 & 1 & 82.42 & 1 & 29.01 & 121 & 34.08 \\
\hline 6 & 77.86 & 6 & 95.85 & 158 & 95.65 & 126 & 56.64 & 7 & 51.99 \\
\hline 74 & 82.59 & 1 & 97.69 & 11 & 97.11 & 6 & 79.18 & 6 & 64.06 \\
\hline 11 & 86.59 & 4 & 98.40 & 82 & 97.72 & 11 & 85.38 & 169 & 73.55 \\
\hline 102 & 88.74 & 11 & 99.07 & 139 & 98.24 & 82 & 89.67 & 49 & 80.98 \\
\hline 1 & 90.68 & 74 & 99.30 & 45 & 98.33 & 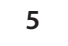 & 91.12 & 164 & 83.33 \\
\hline 202 & 91.67 & 89 & 99.33 & 43 & 98.40 & 124 & 92.88 & 1 & 84.78 \\
\hline-5 & 92.21 & 220 & 99.51 & -4 & 98.48 & 3 & 94.21 & -4 & 85.87 \\
\hline 86 & 92.69 & 7 & 99.61 & 5 & 98.52 & 4 & 95.08 & 5 & 86.47 \\
\hline 41 & 93.12 & 43 & 99.68 & 72 & 98.56 & 43 & 95.85 & 183 & 87.03 \\
\hline 4 & 93.43 & 227 & 99.70 & 3 & 98.59 & 7 & 96.32 & 3 & 87.62 \\
\hline 87 & 93.71 & 45 & 99.72 & 7 & 98.61 & 181 & 96.59 & 11 & 88.07 \\
\hline 43 & 93.96 & 14 & 99.73 & 6 & 98.62 & 24 & 96.72 & 44 & 88.38 \\
\hline 7 & 94.15 & 24 & 99.73 & 24 & 98.63 & 112 & 96.80 & 120 & 88.65 \\
\hline 14 & 94.27 & & & 88 & 98.64 & 102 & 96.86 & 9 & 88.95 \\
\hline 15 & 94.38 & & & & & 45 & 96.90 & 225 & 89.20 \\
\hline 45 & 94.48 & & & & & 15 & 96.93 & & \\
\hline
\end{tabular}

See Table 7 for time intervals. 


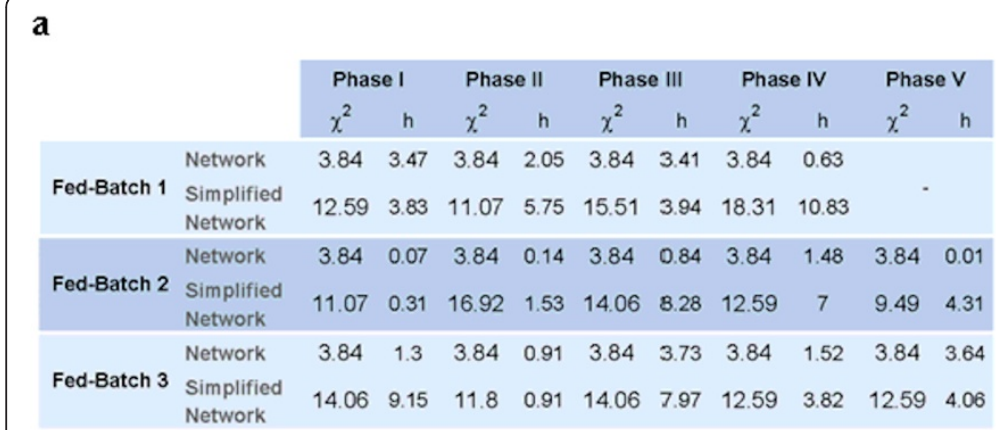

b c

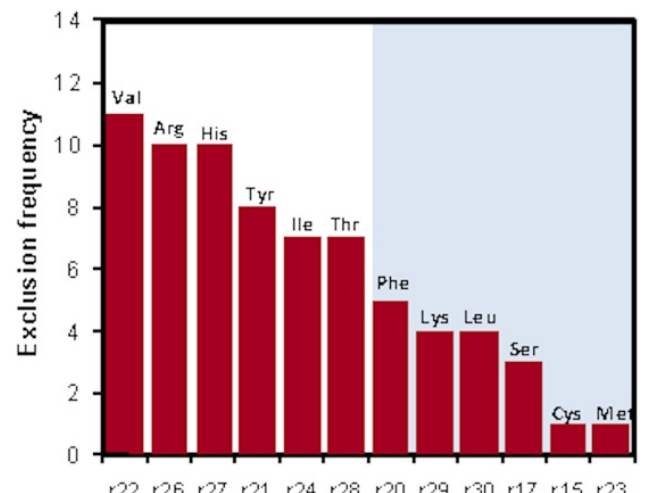

Reactions
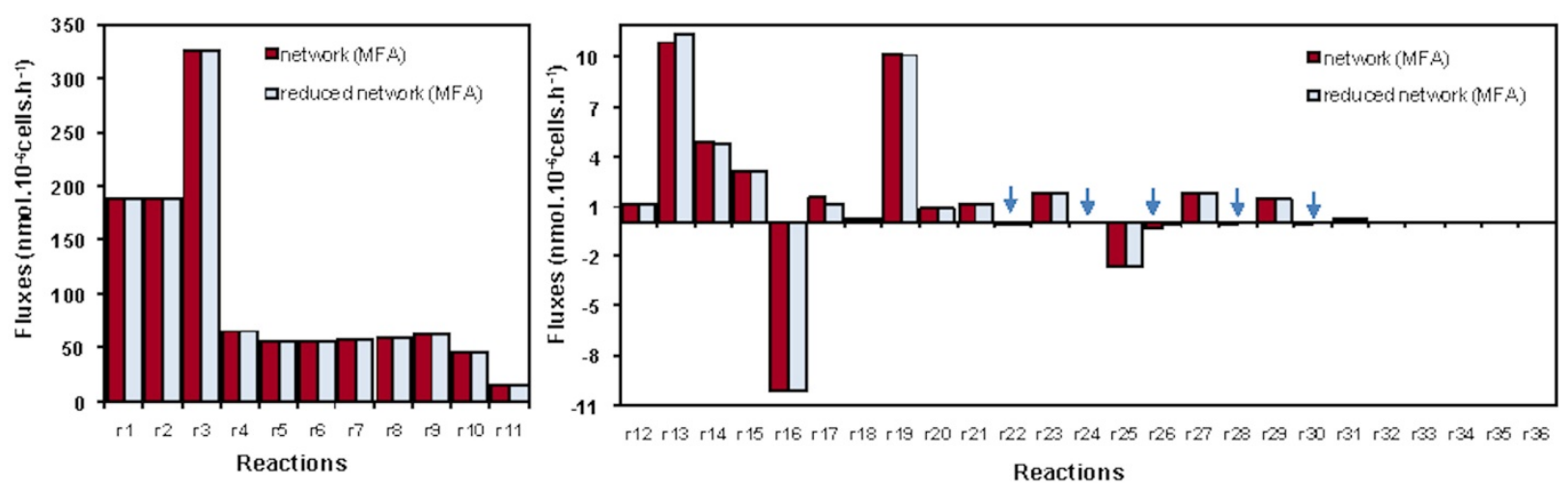

Figure 4 Metabolic network reconstruction based on envirome data. The selected EMs presented in Table 2 and 3 and in Additional File 1 were used to reconstruct reduced metabolic networks for each phase by excluding non-participating reactions. (a) Consistency analysis of metabolic flux estimations. The consistency index $h$ Wang and Stephanopoulos [30], and the $\chi^{2}$ value (with degrees of freedom equal to the number of redundant measurements) are shown for original and simplified networks. Simplified networks have more degrees of freedom since excluding reactions is equivalent to adding null flux constraints. As shown, all phases have a $h$ value lower than the corresponding $\chi^{2}$ value, meaning that the assumed biochemistry is consistent with the measured extracellular rates. (b) Intracellular flux estimations for the first metabolic phase of fed-batch 1. Excluded reactions are indicated with an arrow, showing null or negligible fluxes in the original network. See Figure $2 \mathrm{~b}$ for an overview of all reactions and Table 6 for details. (c) Histogram showing the frequency of reaction exclusion in all metabolic phases of the three fed-batch cultures. These reactions correspond to the catabolism of some amino acids. The ones with low exclusion frequency should be controlled at stoichiometric levels in the medium to avoid ammonia accumulation. For the reactions excluded during each metabolic phase, see Table 6.

method selects a much higher number of EMs than PLP because it does not seek redundancy minimization. The 20 EMs with the highest contribution to flux data variance explained only $66 \%$ of variance (Figure $3 \mathrm{~b}$ ) compared to the $93 \%$ obtained with PLP (Figure 2a). This method also took 111 EMs to reach the 93\% variance obtained with PLP with only 20 EMs. The 105 EMs with weighting factors bigger than 0,1 selected by the Schwartz and Kanehisa [29] method contain the 20 EMs selected by PLP but the former method does not link the EMs with the envirome.

On the whole, these results show that the most significant pathways in cultured mammalian cells could be identified from a larger, redundant set of possible routes using the correlation with the environmental state as the selection criterion. Further, the weighting factors of selected pathways are highly correlated with the environmental state denoting either a causal or effector

Table 6 Excluded reactions by the PLP algorithm during each metabolic phase for fed-batches 1, 2 and 3

\begin{tabular}{lcccc}
\hline & Phase I & Phase II & Phase III & Phase IV \\
\hline Fed-Batch $\mathbf{1}$ & $22,24,26,28,30$ & $17,21,22,26$ & $21,22,24,26,28,29$ & $15,17,20,21,22,23,26,28,29$ \\
Fed-Batch $\mathbf{2}$ & 20, 22, 26, 27 & $17,20,26,27$ & $21,22,24,26,27,28$ & $22,24,27,28,30$ \\
Fed-Batch $\mathbf{3}$ & $21,24,27,29,30$ & $20,22,24,26,27,30$ & $21,22,24,26,27,28$ & $21,22,26,27$ \\
\hline
\end{tabular}


relationship between environmental state and active pathways. Finally, such envirome-correlated pathways explain more than $90 \%$ of flux data variance.

\section{Assessment of pathway variability along culture time}

Pathways may be up- or down-regulated either as a consequence of changes in the environment or driven by changes in the internal state ultimately reflected in a particular environmental "footprint". To monitor such regulatory processes, the PLP algorithm was applied to a moving time window, from the beginning to the end of each fed-batch experiment, to identify EM rearrangements over time. Each culture was partitioned into sequential metabolic phases, where quasi steady state is observed, according to the profiles of extracellular metabolite concentrations.

Table 2 illustrates the highly conserved active pathways obtained during growth in fed-batch 3 (for fedbatches 1 and 2, see Table 4 and 5, respectively). Again, a relatively small number of EMs was able to describe over $90 \%$ of the target rate data in every phase. A moderate degree of pathway conservation amid consecutive phases was observed, with, on average, $42 \%$ of the selected pathways appearing at least twice in each culture. This parallels with $38 \%$ of EMs repeatedly selected in at least two independent experiments (pooling together different phases within each culture), from which we can conclude that cells experience as much metabolic adaptation during culture as between cultures with different pre-set conditions. On the other hand, $65 \%$ of the selected EMs from the combination of all experiments in Figure 2b were conserved between cultures, showing the ability of the method to capture essential common features from independent data sets.

We further analyse in more detail the cases of the reversible EM 4 (Gln $\leftrightarrow$ Glu + Amm) and EM 7 (Asn $\leftrightarrow$ Amm + Asp), linked to amino acids metabolism, which were selected in all phases but at some point changed direction (the minus sign in Table 2). The main factors determining these changes in directionality were the limitation in glutamine concentration (EM 4 turned "negative") and the exhaustion of aspartate (EM 7 turned "positive"). The selection of such pathways (also repeatedly selected in all phases of fed-batch cultures 1 and 2) illustrates the ability of the method to tap on key metabolic adaptation processes.

\section{Consistency analysis of PLP reconstructions}

In order to assess the consistency of PLP results, the discriminated sets of EMs were back-transformed into reduced metabolic networks by excluding reactions not participating in any of the selected pathways. Then, metabolic flux analysis (MFA) was performed to compare intracellular flux distributions of the original BHK metabolic network and PLP reconstructed metabolic networks. Also the consistency index [30] was calculated for each stationary phase. The minimum number of EMs required for obtaining consistency between experimental data and the stoichiometry of simplified networks was the criterion to stop PLP reconstruction. This methodology yielded consistent networks with no more than 17 EMs (Figure 4a). Comparing the fluxes of original and reconstructed networks, it is clear that excluded reactions have fluxes close to zero in the original BHK network, while nonzero fluxes show almost imperceptible differences (for illustration, see Figure $4 \mathrm{~b}$ with fed-batch 1 results).

The excluded reactions (actually pathways of lumped reactions) refer to the catabolism of amino acids (see Table 6), which normally occurs when their concentrations exceed the amount required for proteins synthesis [31]. Some are inactive during the majority of metabolic phases considered, namely r22, r26 and r27 (Figure 4c), corresponding to the catabolism of valine, arginine and histidine, respectively. Therefore, it can be concluded that the consumption of these amino acids is largely determined by protein synthesis (cellular and product), being practically unaffected by their extracellular concentrations within the range present in the medium. On the contrary, the consumption of phenylalanine, lysine, leucine, cysteine and methionine, (r20, r29, r30, r15 and r23, respectively) was largely influenced by their medium concentrations, since the corresponding catabolic pathways were active during more than half of the culture phases, suggesting these amino acids should be provided in controlled, stoichiometric amounts to minimize ammonia formation. A particular scenario arises for serine, whose catabolic conversion to pyruvate (r17) is down-regulated only during three metabolic phases (see Table 6). As mentioned, cells also convert serine into glycine for ammonia detoxification (r16 or EM 6): although maintaining a high extracellular concentration could be beneficial, it may also lead to net ammonia formation if present in excess.

\section{Functional Enviromics Maps (FEM)}

Functional enviromics maps are intended to compile large-scale quantitative information of the interactions between environmental factors and cellular functions. A FEM consists of a data matrix, FEM $=\left\{\mathrm{I}_{\mathrm{i}, \mathrm{j}}\right\}$, with columns representing elementary flux modes, rows representing environmental factors, and $I_{i, j}$ elements the respective regression coefficients. In some cases, the magnitude of $I_{i, j}$ may be interpreted as the strength of up- or down-regulation of a given cellular function $i$ by the environmental factor $j$ (see Methods). The concept of FEM is illustrated in Figure 5 for the case of BHK cells using the full envirome data set, comprising the data of the 6 independent culture experiments. 


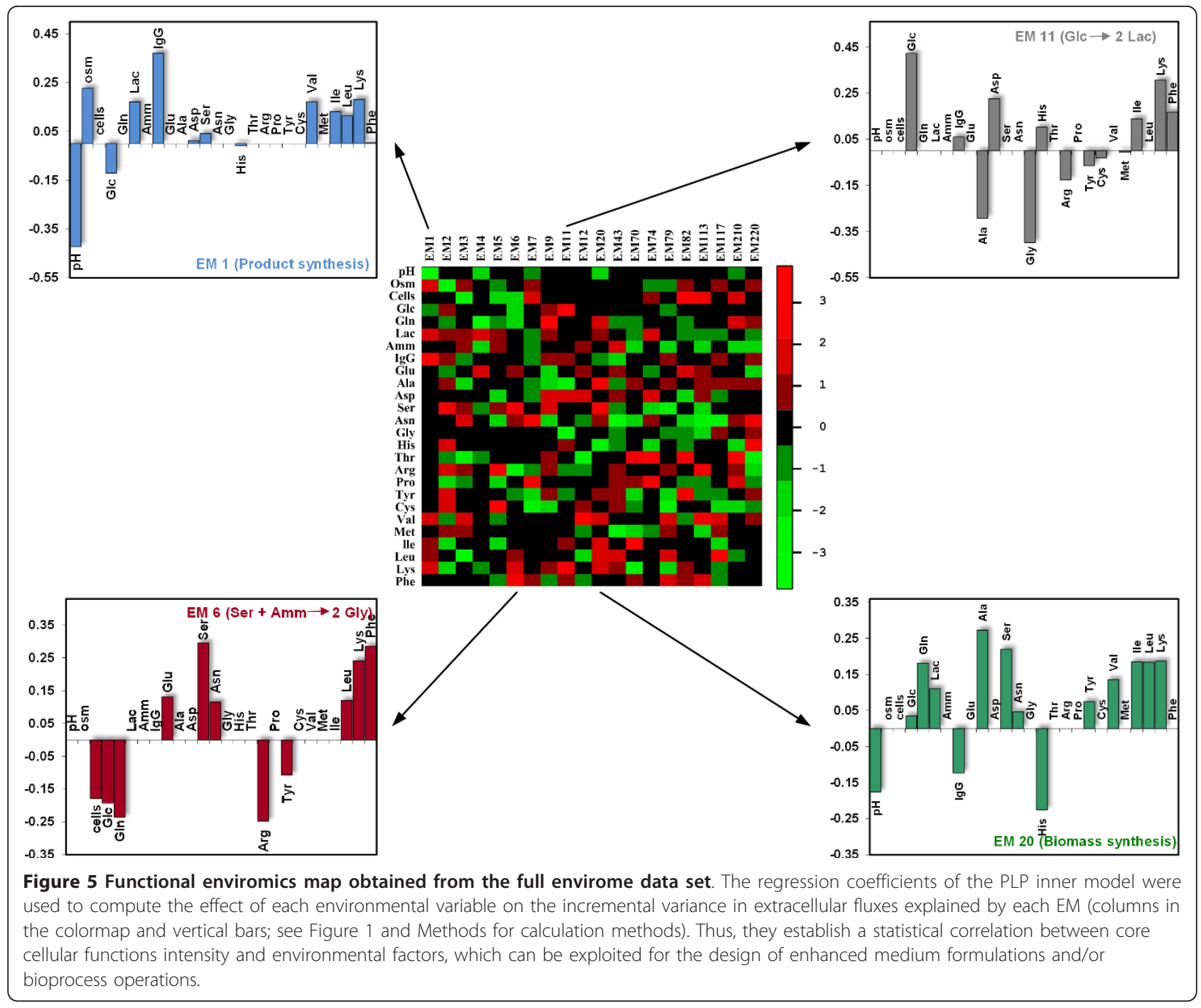

Analysing these data, it can be observed that several envirome factors correlate positively with the flux for biomass synthesis through EM 20, namely the concentrations of glucose, lactate, glutamine and other 8 amino acids. On the other hand, extracellular $\mathrm{pH}$ and the concentrations of product and histidine correlate negatively. While glucose is known to be mandatory for many cell types in culture, BHK cells are capable of glutamine synthesis from glutamate [32]. However, it is also a major energy source for transformed mammalian cell lines, favouring growth when present in the medium. The remaining amino acids correlate positively with this EM since they are more concentrated during initial exponential growth when specific biomass formation is faster. As they are taken up by cells, the cellular division rate decreases as well.

In terms of product synthesis (EM 1), negatively correlated factors include the $\mathrm{pH}$ and glucose concentration, while osmolality and the concentrations of valine, isoleucine, leucine and lysine correlate positively with this flux. The negative correlation with glucose concentration is explained by bioreactor operation factors, as it decreases (or is maintained) along culture time whereas product formation rate increases, rather than by an inhibition mechanism on product synthesis. The effect of $\mathrm{pH}$ deserves further attention, since it also correlates negatively with biomass formation. As described in Methods, the bulk pH was allowed to vary between 7 and 7.25. Some reports agree that maximum growth rates [33] and recombinant protein productivities $[33,34]$ are favoured at $\mathrm{pH}=7$, thus around the lower limit of the values used in this work. On the other hand, it has been shown that specific productivity in mammalian cells is higher with increased bulk osmolality [35-37]. Our results support this observation, however it should be noted that the increase in osmolality resulted mainly from the feeding of 
a concentrated solution during the fed-batch phase. As expected, glucose concentration positively correlated with the flux of lactate formation through EM 11, and serine concentration is the most important factor that activates glycine production through EM 6.

Despite using data from six independent experiments, some environmental factors (namely the concentrations of essential amino acids) show similar variation trends, which precludes disclosure of their true contribution to cellular phenotype. It is clear that the identifiability of environment-EMs relationships is conditional to sufficient "system excitation", which can only be assured by model-based design of experiments. Within proper circumstances of experimental design and physiological range of physical and biochemical variables, the information of EMs regression coefficients can be used to direct the phenotype to a desired state by manipulating genes, envirome or both. It should be noted that EMs represent clusters of genes, thus the EM regression coefficients translate the lumped effect of kinetic and genetic regulation induced by the envirome.

\section{Conclusions}

While in functional genomics the aim is to unravel gene functions from the analysis of transcriptome and proteome dynamical data [38-40], here we propose a reverse, envirome-to-function analysis approach sourced by dynamic envirome data. This approach is founded on the assumption that the genome sets the universe of cellular functions while the envirome sets the relative contribution of each function to the observed phenotype. We have developed a systems level methodology that deconvolutes the observed phenotype (i.e. fluxome) into gene dependent structures (the structure of elementary flux modes) and envirome dependent structures (the relative weight of elementary flux modes ). Then, only the envirome dependent structures are linearly regressed against envirome components to discriminate the core cellular functions that correlate with the environmental state.

When applied to a recombinant BHK cell line, environmental data finds correspondence to less than 20 elementary flux modes, while explaining over $90 \%$ of flux variance. Most of the discriminated elementary flux modes are typical routes known to be active in cultured mammalian cells, while excluded routes are related with the catabolism of those amino acids not in excess in the medium, thus being directly used for protein synthesis. Furthermore, metabolic flux distributions of reduced metabolic networks, accounting for the discriminated core cellular functions only, were shown to be consistent with metabolic flux distributions of the original, unreduced metabolic network. These results essentially show that through the life of the culture, phenotypic variability is almost completely traceable by monitoring changes in envirome data. The remaining $10 \%$ of unexplained variance corresponds mostly to experimental error, thus leaving little room for genetically induced variability that cannot be traced to envirome data. On the whole, such results support the feasibility of a function reconstruction approach guided by the collection and analysis of envirome data sets.

When applied to a sliding time window along a single culture experiment, it was observed that several discriminated EMs are not active during all culture phases while others may change direction at some point. Such short-term dynamic changes correspond to metabolic adaptation induced by environmental stimuli. While some metabolic adaptation effects are readily interpretable in terms of exhaustion or limitation of essential substrates, some other have nontrivial interpretation and may correspond to unknown functions of environmental factors. The analysis of EM rearrangements can provide insights about the mechanisms underlying such metabolic adaptation. Despite the variability in observed EMs with culture time, a high degree of conservation of cellular function among the six different cultivation experiments was obtained (65\% of discriminated EMs appear in all cultures). These observations support the results presented by Ma et al. [22] who used computational methods to identify network topologies that can achieve biochemical adaptation. They concluded that despite the diversity of possible biochemical networks, only a finite set of core topologies represent a robust adaptation response to a stimulus. These results support the hypothesis of a minimal set of core cellular functions activated by the envirome. The use of less informative envirome data sets (when compared to metabolome, proteome or transcriptome data sets) translates into lower discriminating power of cellular functions, particularly of those cellular functions producing similar environmental footprints. However, since only a small number of core cellular functions with distinctive footprints are active they are easily identified from an analysis of the envirome, thus constituting a vital support of cell functional enviromics.

While our intention was to demonstrate the principle of cell functional enviromics, its full potential can be realized when large-scale, high-throughput analysis techniques are employed for envirome-wide reconstruction of cellular functions eventually leading to high resolution functional enviromics maps. This approach has many interesting practical applications ranging from drug design to macroscopic process control. A point to be made is that finding a statistical correlation, even if linked with a metabolic structure, does not allow one to discriminate between a "cause" and an "effect". Such discrimination is only possible after a systematic analysis 
on the basis of a sound experimental design, which might include simultaneous environmental and genetic perturbations.

Fundamental issues, which remain to be clarified, include the degree of conservation of envirome functions among different species. One can anticipate that, given the role of environmental factors in the evolution of life, some degree of conservation may exist between species, as implicitly acknowledged by those who defend the reverse ecological principle [7]. A systematic functional enviromics study applied to different species and genotype variants could shed light on this issue.

The need for envirome-driven systems biology approaches to address human diseases has long been recognized [41]. Others have proposed enviromics as a complement to genomic and proteomic studies of mental health [42]. Even the notion of 'functional enviromics' has been set forth as a counterpart to functional genomics in tackling schizophrenia disorders [43]. Our approach has been to explore this concept in cellular systems- cell functional enviromics - which we view as a natural step in both fundamental and applied molecular biology research.

\section{Methods}

\section{Envirome-guided projection to latent pathways (PLP)}

According to the definition of elementary flux modes, the universe of infinite flux solutions, $r$, of a given metabolic network operating in steady state can be described as a non-negative weighted sum of a finite number of elementary flux modes, $e_{\mathrm{i}}$ (e.g. $\left.[12,14,15]\right)$ :

$$
r=\lambda_{1} \cdot e_{1}+\lambda_{2} \cdot e_{2}+\cdots+\lambda_{n e m} \cdot e_{n e m}, \quad \forall_{r}
$$

Elementary modes thus represent unique and nondecomposable flux solutions, from which all possible system solutions can be obtained through the proper determination of elementary modes weighting factors, $\lambda_{\mathrm{i}}$. Several methods were proposed to calculate weighting factors, $\lambda_{i}$, from flux vectors, r. (e.g. [27-29]). As noted by Wiback et al. [27], the reconstruction of a particular flux vector according to Eq. (1) is not unique if the number of elementary modes is higher than the number of fluxes, which is generally the case. Unique solutions can however be obtained with additional assumptions about the biological system, such as the maximisation of number of elementary modes [28], or minimisation of the values of weighting factors to select the elementary modes that are closest to the actual biological state [29]. In the present study we also calculate a unique solution for the weighting factors $\lambda_{i}$ although under completely different criteria. We set the weighting factors $\lambda_{i}$ as linear functions of envirome variables, $\mathrm{x}(\mathrm{t})$, which represents additional measured information in our method that is not used in other methods. The objective function in our case consists of:

i) maximising correlation between $\lambda_{i}$ and envirome variables (or equivalently between $\mathrm{r}(\mathrm{t}$ ) and $\mathrm{x}(\mathrm{t})$ ), and

ii) minimising redundancy, i.e. eliminating all elementary modes with weak correlations with the envirome.

The basic idea is that the genome sets the structure of the elementary flux modes, while the relative weights of elementary flux modes are to a large extent set by the environment.

According to the above criteria, the PLP algorithm was designed to maximise the covariance between an input data matrix, $\mathrm{X}=\{\mathrm{x}(\mathrm{t})\}$, of independent envirome observations, $x(t)$, and an output response matrix, $R=\{r$ $(\mathrm{t})\}$, of observed steady state reaction rates, $\mathrm{r}(\mathrm{t})$, under the constraint of a plausible set of elementary flux modes. This problem can be expressed mathematically by the following constrained linear program:

$$
\begin{array}{cc}
\text { Maximize } & \operatorname{cov}(X, R) \\
\text { s.t. } & \left\{\begin{array}{l}
R=\Lambda \times E^{T} \\
\Lambda=X \times C^{T}
\end{array}\right.
\end{array}
$$

with $X=\{x(t)\}$ a $n p \times n x$ matrix of $n p$ independent observations of envirome vectors $x(t)(\operatorname{dim}(x)=n x), R=$ $\{\mathrm{r}(\mathrm{t})\}$ a $\mathrm{np} \times \mathrm{nr}$ matrix of $\mathrm{np}$ independent observations of reaction rates, $\mathrm{r}(\mathrm{t})(\operatorname{dim}(\mathrm{r})=\mathrm{nr}), \mathrm{E}=\left\{\mathrm{e}_{\mathrm{i}}\right\} \mathrm{a} \mathrm{nr} \times \mathrm{nem}$ matrix of nem elementary flux modes, $e_{i}\left(\operatorname{dim}\left(e_{i}\right)=n r\right)$ (for the BHK metabolic network, $\mathrm{E}$ is the $24 \times 251$ matrix given in the Additional File 1$), \Lambda=\{\lambda(\mathrm{t})\}$ a $\mathrm{np} \times$ nem matrix of weighting vectors, $\lambda(t)$, of elementary flux modes $(\operatorname{dim}(\lambda)=$ nem $)$ and $C$ a nem $\times$ nx matrix of regression coefficients.

Maximising covariance implies two concomitant goals, namely maximising variance and minimising redundancy. Unconstrained maximisation of covariance can be achieved by the very popular projection to latent structures method, also known as partial least squares (PLS). Since PLP is derived from PLS, in the lines below we first review the structure of PLS and then show how it can be modified to PLP.

PLS is a multivariate linear regression technique that maximises the covariance between an input matrix, $X$, called the predictor, and an output response matrix, which here is the target rate data, $R$. It differs from traditional multivariate linear regression in that it decomposes iteratively both the predictor and the response matrices into a reduced set of uncorrelated latent variables, thereby eliminating redundancy in the input and output data sets. More specifically, the matrices $X$ and $R$ are decomposed into loadings matrices $(W$ and $Q$ ), 
scores matrices ( $T$ and $U)$ and residuals matrices $\left(E_{X}\right.$ and $\left.E_{R}\right)$ :

$$
\begin{aligned}
& X=T \times W^{T}+E_{X} \\
& R=U \times Q^{T}+E_{R}
\end{aligned}
$$

The columns of the loadings matrices $W$ and $Q$ are the latent variables in which the input and output matrices are decomposed, respectively. Additionally, the outputs scores matrix $\mathrm{U}$ is linearly regressed against the inputs scores matrix $\mathrm{T}$ :

$$
U=T \times B^{T}+E_{U}
$$

where $B$ holds the regression coefficients determined by minimising the residuals $E_{U}$. Finally, the explained variance in target data (which in our case is flux data, $\mathrm{R})$ by the model is given by the following equation:

$$
\operatorname{var}(\%)=100 \times\left(1-\frac{\sum_{i} \sum_{j} E_{R i, j}{ }^{2}}{\sum_{i} \sum_{j} R_{i, j}{ }^{2}}\right)
$$

with indexes $i$ and $j$ denoting observation and flux indexes respectively. Solving system Eqs. (3a-c), which imply minimising residuals $E_{X}$. $E_{R}$ and $E_{U}$, can be effectively performed by the very popular Non Iterative Partial Least Squares (NIPALS) algorithm. For more details about PLS and NIPALS see, for instance, Geladi and Kowalski [44].

PLP can be viewed as a constrained version of PLS by the inclusion of the elementary flux modes constraints. Given their equivalent mathematical structures, an interesting comparison may be made between projection of target reaction rate data, $\mathrm{R}$, onto latent variables $\mathrm{Q}$ (performed in PLS according to Eq. (3b)), and projection of flux vectors onto elementary modes weighting factors according to Eq. (1). Indeed, elementary flux modes $e_{i}$ can be viewed as PLS latent variables equivalents, while the elementary modes weighting factors $\lambda_{i}$ can be interpreted as PLS latent variables score values. Following this analogy, a straightforward modification to Eq. (3b) can be introduced

$$
R=U \times E^{T}+E_{R}
$$

by substituting PLS loading matrix, Q, by the elementary modes matrix, E. This means that the latent structures $E$ are no longer degrees of freedom in PLP as $Q$ was in PLS. Instead, $E$ represents a constraint to the relationship between $\mathrm{X}$ and $\mathrm{R}$ imposed by the metabolic network structure and ultimately by the genome of the cells.

An advantage of PLP over PLS is that both the latent variables and score values of the target matrix have in
PLP a physical meaning. The latent variables are latent pathways while scores $U$ are equivalent to $\Lambda$, i.e. they represent the relative weighting factors of latent pathways. For this reason, the algorithm is called projection to latent pathways. Moreover, the regression coefficients $\mathrm{B}$ can be used to deduce the functional enviromics matrix, FEM, as follows:

$$
F E M=W \times B
$$

FEM is a $\mathrm{nx} \times$ nem matrix comprising the regression coefficients of elementary flux modes against envirome components, thus providing information of how elementary flux modes are up- or down-regulated by envirome components.

The PLP algorithm was coded in MATLAB (Mathworks, Inc) as a modified version of the NIPALS algorithm \{Geladi, $1986 \# 15\}$, wherein the loadings of the outputs are fixed a priori according to the elementary modes structure, $E$. The calculation of the elementary modes is not automatically integrated in PLP. For that we used the METATOOL 5.0 [13] as explained below.

\section{Cell line and culture conditions}

A BHK-21 cell line constitutively expressing the fusion glycoprotein IgG1-IL2 (antibody type one linked to Interleukin two) was used in this study [45]. Six cell cultures were performed in $2 \mathrm{~L}$ bioreactor vessels in a serum-free and protein-free medium, SFM4CHO (Hyclone), without glucose and glutamine. One culture was performed in batch mode and five in fed-batch mode. The starting volume was $950 \mathrm{ml}$, dissolved oxygen was kept at $30 \%$ of air saturation through air/nitrogen sparging at a gas flow rate of $0.02 \mathrm{vvm}$; the agitation rate was kept at $70 \mathrm{rpm}$. The $\mathrm{pH}$ was controlled between 7 and 7.25 by addition of $\mathrm{NaOH}(0.2$ $\mathrm{mM})$ or $\mathrm{CO}_{2}$. The batch culture started at $48 \mathrm{mM}$ of glucose, $1.2 \mathrm{mM}$ of Glutamine, $2.8 \mathrm{mM}$ of Glutamate, $1.3 \mathrm{mM}$ of Serine and $0.75 \mathrm{mM}$ of Aspartate. As for the fed-batch cultures, the initial concentrations and feeding strategies are given in Table 1.

\section{Envirome profiling}

27 envirome components were profiled, namely temperature, $\mathrm{pH}$, osmolality and concentrations of 24 extracellular compounds determined as follows. Cell counts were determined in a Fuchs-Rosenthal haemocytometer; cell viability was assessed by the trypan blue dye exclusion method. Product concentration was determined by ELISA (see Teixeira et al. [46] for details). The concentrations of the main nutrients (glucose (Glc), glutamine (Gln), glutamate (Glu) and lactate (Lac)) were determined enzymatically using the biochemical analyser YSI 7100 (Yellow Springs, USA). The osmolality of culture 
Table 7 Observed extracellular rates for several metabolic phases within three fed-batch cultures

\begin{tabular}{|c|c|c|c|c|c|c|c|c|c|c|c|c|c|c|}
\hline \multirow[b]{2}{*}{$\mathbf{R}_{\text {obs }}$} & \multicolumn{4}{|c|}{ Fed-batch 1} & \multicolumn{5}{|c|}{ Fed-batch 2} & \multicolumn{5}{|c|}{ Fed-batch 3} \\
\hline & $\begin{array}{c}\text { Ph I } \\
(21-45 \text { h) }\end{array}$ & $\begin{array}{c}\mathrm{Ph} \mathrm{II} \\
(45-70 \mathrm{~h})\end{array}$ & $\begin{array}{c}\text { Ph III } \\
(70-95 \text { h) }\end{array}$ & $\begin{array}{c}\text { Ph IV } \\
(95-118 \text { h) }\end{array}$ & $\begin{array}{c}\mathrm{Ph} \mathrm{I} \\
(25-50 \mathrm{~h})\end{array}$ & $\begin{array}{c}\text { Ph II } \\
(50-70 \text { h) }\end{array}$ & $\begin{array}{c}\text { Ph III } \\
(70-90 \text { h) }\end{array}$ & $\begin{array}{c}\text { Ph IV } \\
(90-110 \text { h) }\end{array}$ & $\begin{array}{c}\text { Ph V } \\
(110-135 \text { h) }\end{array}$ & $\begin{array}{c}\mathrm{Ph} \mathrm{I} \\
(50-75 \mathrm{~h})\end{array}$ & $\begin{array}{c}\mathrm{Ph} \mathrm{II} \\
(75-100 \text { h) }\end{array}$ & $\begin{array}{c}\text { Ph III } \\
(100-125 \text { h) }\end{array}$ & $\begin{array}{c}\text { Ph IV } \\
(125-150 \text { h) }\end{array}$ & $\begin{array}{c}\text { Ph V } \\
(150-175 \text { h) }\end{array}$ \\
\hline$\mu$ & 0.019 & 0.022 & 0.022 & 0.012 & 0.020 & 0.020 & 0.018 & 0.014 & 0.009 & 0.020 & 0.017 & 0.014 & 0.009 & 0.008 \\
\hline rGlc & -190.33 & -76.60 & -71.99 & -50.89 & -187.25 & -88.71 & -74.53 & -78.89 & -51.89 & -99.03 & -71.38 & -99.38 & -63.53 & -75.45 \\
\hline rGln & -8.74 & -3.56 & -5.24 & -4.31 & -10.23 & -7.39 & -2.02 & -1.85 & -1.20 & -12.46 & -3.73 & -2.85 & -1.47 & -1.62 \\
\hline rLac & 324.81 & 68.80 & 19.97 & 8.95 & 206.02 & 116.56 & 76.71 & 59.78 & 46.56 & 94.97 & 32.10 & 59.04 & 24.52 & 17.45 \\
\hline rAmm & 9.85 & 12.94 & 10.50 & 9.68 & 16.32 & 12.75 & 9.88 & 8.17 & 6.34 & 9.83 & 9.40 & 12.91 & 14.04 & 12.47 \\
\hline rlgG & 0.000215 & 0.000326 & 0.000291 & 0.000224 & 0.000167 & 0.000257 & 0.000301 & 0.000308 & 0.000264 & 0.000214 & 0.000280 & 0.000317 & 0.000368 & 0.000465 \\
\hline rGlu & 3.90 & -8.26 & -15.43 & -14.25 & 5.86 & -9.97 & -14.42 & -18.72 & -13.34 & -3.49 & -9.18 & -12.19 & -10.96 & -13.61 \\
\hline rAla & 0.13 & -2.63 & -2.83 & -1.91 & 0.45 & -2.34 & -3.00 & -2.27 & -1.54 & -1.65 & -4.23 & -4.16 & -1.93 & -0.56 \\
\hline rAsp & -15.89 & -14.38 & -8.20 & -0.54 & -12.71 & -9.51 & -2.22 & -0.50 & 0.01 & -5.96 & -4.52 & -4.34 & -2.93 & -2.33 \\
\hline rSer & -19.18 & -16.59 & -14.63 & -3.06 & -13.63 & -10.89 & -13.84 & -14.43 & -7.80 & -15.58 & -11.79 & -10.03 & -5.69 & -4.47 \\
\hline rAsn & 2.91 & -3.98 & -3.24 & -2.31 & -3.30 & -3.05 & -2.55 & -1.86 & -1.45 & 0.30 & -2.37 & -3.96 & -2.96 & -2.70 \\
\hline rGly & 13.83 & 7.17 & 1.26 & -0.01 & 9.50 & 7.34 & 6.18 & 5.84 & 5.16 & 8.53 & 6.30 & 3.66 & 1.93 & 0.47 \\
\hline rHis & -3.17 & -2.80 & -1.67 & 0.87 & -1.08 & -1.10 & -0.65 & -0.67 & -0.47 & -2.53 & -0.78 & -0.73 & -0.52 & -0.56 \\
\hline rThr & -3.30 & -3.65 & -3.47 & -1.79 & -2.99 & -2.95 & -2.53 & -1.74 & -1.30 & -2.58 & -2.10 & -2.47 & -1.37 & -1.33 \\
\hline rArg & -3.15 & -2.88 & -3.38 & -1.93 & -2.88 & -2.88 & -2.55 & -2.52 & -1.41 & -2.69 & -2.35 & -2.27 & -1.47 & -1.26 \\
\hline rPro & -5.84 & -5.27 & -1.81 & -1.00 & -5.82 & -2.93 & -0.83 & 0.90 & 1.60 & 9.34 & 2.41 & -1.07 & -0.39 & -0.09 \\
\hline rTyr & -1.75 & -1.35 & -1.56 & -1.13 & -1.97 & -1.41 & -0.58 & -0.39 & -0.21 & -1.17 & -1.13 & -1.02 & -0.75 & -1.08 \\
\hline rCys & -2.97 & -2.58 & -1.59 & -0.88 & -3.03 & -1.45 & -2.98 & -3.34 & -1.84 & -3.36 & -2.63 & -2.54 & -1.69 & -1.41 \\
\hline rVal & -3.51 & -3.84 & -3.68 & -2.04 & -2.96 & -2.82 & -2.53 & -2.20 & -1.40 & -2.56 & -2.03 & -2.50 & -1.95 & -1.77 \\
\hline rMet & -3.06 & -4.76 & -2.46 & -0.72 & -2.35 & -1.89 & -1.35 & -0.82 & -0.50 & -1.68 & -1.06 & -1.46 & -1.21 & -1.32 \\
\hline rlle & -2.52 & -3.16 & -2.59 & -1.54 & -2.89 & -2.40 & -1.99 & -1.41 & -1.12 & -1.93 & -1.59 & -1.68 & -1.68 & -1.47 \\
\hline rLeu & -4.66 & -5.52 & -5.11 & -2.67 & -5.01 & -4.51 & -3.47 & -2.90 & -1.85 & -3.83 & -3.07 & -4.68 & -3.29 & -2.74 \\
\hline rLys & -5.62 & -4.46 & -4.27 & -2.32 & -5.25 & -4.08 & -3.35 & -2.59 & -2.07 & -3.31 & -3.62 & -4.77 & -2.96 & -1.65 \\
\hline rPhe & -2.69 & -2.27 & -1.83 & -0.75 & -1.70 & -1.60 & -1.94 & -3.25 & -1.03 & -0.98 & -0.93 & -0.98 & -0.79 & -0.80 \\
\hline
\end{tabular}

Units are in nmol. $\left(10^{6} \text { cells.h }\right)^{-1}$. A minus sign indicates consumption. $\mu$ refers to the specific growth rate $\left(\mathrm{h}^{-1}\right)$, calculated using the concentrations of viable cells 
supernatant was assayed using a Digital Micro-Osmometer, Type 5R (Hermann Roebling Messtechnik, Germany). Amino acids concentrations were determined by high performance liquid chromatography (HPLC) using a protocol optimized in our Lab (for details, see Carinhas et al. [47]). Ammonia concentrations were determined enzymatically using an UV assay (Boehringer Manheim, R-Biopharm AG, Germany).

\section{BHK Metabolic network}

The BHK metabolic network adopted in this study comprised 57 metabolic reactions (including transport/diffusion across the plasma membrane), 35 intracellular metabolites and 24 extracellular metabolites and other compounds. Considered reactions explain the major fluxes of carbon and nitrogen, namely glycolysis, glutaminolysis, TCA cycle, pentose-phosphate pathway, recombinant product synthesis and biosynthesis of cellular components (nucleotides, carbohydrates, lipids and proteins). The catabolism of all amino acids except tryptophan is also considered. The network was simplified by lumping series of metabolic reactions, free of branching points, without loss of representativeness under steady-state. A detailed description can be found in Additional File 2.

\section{Metabolic flux analysis}

For metabolic flux analysis (MFA), the difference between the number of reactions and the number of intracellular metabolites is 57-35 $=22$, which means that at least 22 measured fluxes are required to obtain a determined MFA system. Envirome profiling provided data of 24 exchange fluxes, which means that the MFA system becomes redundant with 24-22 = 2 degrees of freedom. As such, the full vector of 57 fluxes was partitioned into known (extracellular) and unknown (intracellular) subsets of 24 and 33 fluxes respectively. Measured fluxes for different phases of fed-batch cultures are presented as Table 7 . The resulting redundant system of linear equations was solved by the weighted least squares method [48]. The error standard deviation of measured fluxes were $5 \%$ for glucose, lactate, glutamine, glutamate and ammonia concentrations, 10\% for biomass, product and the remaining amino acids concentrations. All MFA calculations were performed using the FluxAnalyzer software [13].

\section{Consistency analysis of metabolic flux distributions}

Given that the MFA system is redundant with two degrees of freedom, the consistency index method [30] can be applied to verify consistency of calculated metabolic flux distributions in relation to assumed biochemistry and steady state assumption. Briefly, the consistency index, $h$, was calculated according to the method described in Wang and Stephanopoulos [30]. Then, the values of $h$ were compared to the $\chi^{2}$ statistical test for two degrees of freedom. Whenever $h$ is below the $\chi^{2}$ threshold value the system is said to be consistent. These tasks were performed using FluxAnalyzer [13].

\section{Determination of BHK elementary flux modes}

The BHK metabolic network was firstly manipulated according to the guidelines in Gagneur and Klamt [49], and then the respective EMs were computed using FluxAnalyzer [13]. This medium scale network has a total of 251 EMs, which represent, in the context of the present study, the universe of cellular functions to be screened against envirome components. Among the full set of 251 EMs, 139 refer to biomass synthesis. A closer look to these EMs reveals that different by-products at different stoichiometric ratios are secreted or excreted concomitantly with biomass formation, namely ammonia, lactate, glutamate, alanine, aspartate and/or glycine, thus producing a distinctive environmental footprint that can be used for their discrimination. It should be noted that a single EM describes product synthesis since the underlying synthesis reaction involves only unbalanced amino acids (the carbohydrate content of the fusion glycoprotein was not considered). Additional File 1 provides a complete list of the 251 BHK elementary flux modes, formulated in terms of extracellular compounds stoichiometry.

\section{Application of PLP to BHK data}

For the present BHK problem, the input data set $\mathrm{X}$ comprises the above enumerated 27 envirome factors. The target data-set $\mathrm{R}$ comprises measurements of the same 24 exchange fluxes used for MFA. The universe of EMs is formed by the 251 EMs obtained for BHK (see Additional File 1), The subset of EMs with significant correlation with the envirome is shown in Table 3.

\section{Additional material}

Additional file 1: BHK elementary modes. List of elementary modes obtained from the BHK metabolic network (additional file 2). Elementary modes are represented in reduced form in terms of extracellular metabolites.

Additional file 2: BHK metabolic network. Biochemical reactions/ pathways, enzymes and biomass composition considered in the metabolic model of BHK cells

\section{Acknowledgements}

Financial support for this work was provided by the Portuguese Fundação para a Ciência e Tecnologia through project PTDC/EBB-EBI/103761/2008, PhD grant SFRH/BD/36676/2007 (NC) and Post-Doc grant SFRH/BPD/46277/ 2008 (JD). We would like to acknowledge Professor Michael Semmens from 
the University of Minnesota for his valuable assistance in reviewing this manuscript.

\section{Author details}

${ }^{1}$ Instituto de Tecnologia Química e Biológica - Universidade Nova de Lisboa (ITQB-UNL), Av. República, Quinta do Marquês, 2781-901 Oeiras, Portugal. ${ }^{2}$ Instituto de Biologia Experimental e Tecnológica (IBET), Av. República, Quinta do Marquês, 2781-901 Oeiras, Portugal. ${ }^{3}$ REQUIMTE, Systems Biology \& Engineering Group, Departamento de Química, Faculdade de Ciências e Tecnologia, Universidade Nova de Lisboa (FCT-UNL), 2829-516 Caparica, Portugal.

\section{Authors' contributions}

The project was conceived by $\mathrm{RO}$ and experiments planned by AT, RO, PA and MJTC. Experiments were performed by AT and NC. MS and AC supervised the bioreaction and JC developed software to support fed-batch control. PLP algorithm was developed by JD, MVS and RO. Data analysis and manuscript writing were performed by AT, JD, NC and RO. All authors read and approved the final manuscript.

Received: 12 January 2011 Accepted: 6 June 2011

Published: 6 June 2011

\section{References}

1. Hunter DJ: Gene-environment interactions in human diseases. Nat Rev Genet 2005, 6(4):287-298.

2. Sauer U: High-throughput phenomics: experimental methods for mapping fluxomes. Curr Opin Biotech 2004, 15(1):58-63.

3. Smith EN, Kruglyak L: Gene-environment interaction in yeast gene expression. Plos Biol 2008, 6(4):810-824.

4. Kyoto Encyclopedia of Genes and Genomes (KEGG). [http://www genome.jp/kegg/].

5. BioCyc Database Collection. [http://biocyc.org/]

6. Janga SC, Babu MM: Network-based approaches for linking metabolism with environment. Genome Biol 2008, 9(11):1-5.

7. Borenstein $E$, Kupiec M, Feldman MW, Ruppin E: Large-scale reconstruction and phylogenetic analysis of metabolic environments. P Natl Acad Sci USA 2008, 105(38):14482-14487.

8. Pal C, Papp B, Lercher MJ: Adaptive evolution of bacterial metabolic networks by horizontal gene transfer. Nat Genet 2005, 37(12):1372-1375.

9. Kreimer A, Borenstein E, Gophna U, Ruppin E: The evolution of modularity in bacterial metabolic networks. P Natl Acad Sci USA 2008 105(19):6976-6981

10. Allen J, Davey HM, Broadhurst D, Heald JK, Rowland JJ, Oliver SG, Kell DB: High-throughput classification of yeast mutants for functional genomics using metabolic footprinting. Nat Biotechnol 2003, 21(6):692-696.

11. Kell DB, Brown M, Davey HM, Dunn WB, Spasic I, Oliver SG: Metabolic footprinting and systems biology: the medium is the message. Nat Rev Microbiol 2005, 3(7):557-565.

12. Klamt S, Stelling J: Two approaches for metabolic pathway analysis? Trends Biotechnol 2003, 21(2):64-69.

13. Klamt S, Stelling J, Ginkel M, Gilles ED: FluxAnalyzer: exploring structure, pathways, and flux distributions in metabolic networks on interactive flux maps. Bioinformatics 2003, 19(2):261-269.

14. Palsson BO, Price ND, Papin JA: Development of network-based pathway definitions: the need to analyze real metabolic networks. Trends Biotechnol 2003, 21(5):195-198.

15. Papin JA, Stelling J, Price ND, Klamt S, Schuster S, Palsson BO: Comparison of network-based pathway analysis methods. Trends Biotechnol 2004, 22(8):400-405.

16. Schuster S, Dandekar T, Fell DA: Detection of elementary flux modes in biochemical networks: a promising tool for pathway analysis and metabolic engineering. Trends Biotechnol 1999, 17(2):53-60.

17. Schuster S, Fell DA, Dandekar T: A general definition of metabolic pathways useful for systematic organization and analysis of complex metabolic networks. Nat Biotechnol 2000, 18(3):326-332.

18. Wagner $C$ : Nullspace approach to determine the elementary modes of chemical reaction systems. J Phys Chem B 2004, 108(7):2425-2431.

19. Forster J, Gombert AK, Nielsen J: A functional genomics approach using metabolomics and in silico pathway analysis. Biotechnol Bioeng 2002, 79(7):703-712.
20. Pachkov M, Dandekar T, Korbel J, Bork P, Schuster S: Use of pathway analysis and genome context methods for functional genomics of Mycoplasma pneumoniae nucleotide metabolism. Gene 2007, 396(2):215-225.

21. Klamt S, Gagneur J, von Kamp A: Algorithmic approaches for computing elementary modes in large biochemical reaction networks. IET Syst Biol 2005, 152(4):249-255

22. Ma WZ, Trusina A, El-Samad H, Lim WA, Tang C: Defining network topologies that can achieve biochemical adaptation. Cell 2009, 138(4):760-773.

23. Gould GW, Holman GD: The glucose-transporter family - structure, function and tissue-specific expression. Biochem J 1993, 295:329-341.

24. Wlaschin KF, Hu WS: Engineering cell metabolism for high-density cell culture via manipulation of sugar transport. J Biotechnol 2007, 131(2):168-179.

25. Cruz HJ, Ferreira AS, Freitas CM, Moreira JL, Carrondo MJT: Metabolic responses to different glucose and glutamine levels in baby hamster kidney cell culture. Appl Microbiol Biot 1999, 51(5):579-585.

26. Jeong $\mathrm{YH}$, Wang SS: Role of glutamine in hybridoma cell-culture - effects on cell-growth, antibody-production, and cell-metabolism. Enzyme Microb Tech 1995, 17(1):47-55.

27. Wiback SJ, Mahadevan R, Palsson BO: Reconstructing metabolic flux vectors from extreme pathways: defining the alpha-spectrum. J Theor Biol 2003, 224(3):313-324

28. Nookaew I, Meechai A, Thammarongtham C, Laoteng K, Ruanglek V Cheevadhanarak S, Nielsen J, Bhumiratana S: Identification of flux regulation coefficients from elementary flux modes: A systems biology tool for analysis of metabolic networks. Biotechnol Bioeng 2007, 97(6):1535-1549.

29. Schwartz JM, Kanehisa M: Quantitative elementary mode analysis of metabolic pathways: the example of yeast glycolysis. BMC Bioinformatics 2006, 7:20

30. Wang NS, Stephanopoulos G: Application of macroscopic balances to the identification of gross measurement errors. Biotechnol Bioeng 1983, 25(9):2177-2208

31. Me LZ, Zhou WC: Fed-batch cultivation of mammalian cells for the production of recombinant proteins. In Cell Culture Technology for Pharmaceutical and Cell-Based Therapies. Edited by: Ozturk S, Hu W-S. Boca Raton: Crc Press-Taylor 2006:349-386.

32. Christie A, Butler M: The adaptation of BHK cells to a non-ammoniagenic glutamate-based culture medium. Biotechnol Bioeng 1999, 64(3):298-309.

33. Yoon SK, Choi SL, Song JY, Lee GM: Effect of culture pH on erythropoietin production by chinese hamster ovary cells grown in suspension at 32.5 and 37.0 degrees C. Biotechnol Bioeng 2005, 89(3):345-356.

34. Osman JJ, Birch J, Varley J: The response of GS-NSO myeloma cells to $\mathrm{pH}$ shifts and pH perturbations. Biotechnol Bioeng 2001, 75(1):63-73.

35. Lin JQ, Takagi M, Qu YB, Gao PJ, Yoshida T: Enhanced monoclonal antibody production by gradual increase of osmotic pressure. Cytotechnology 1999, 29(1):27-33.

36. Ozturk SS, Palsson BO: Effect of medium smolarity on hybridoma growth, metabolism, and antibody-production. Biotechnol Bioeng 1991, 37(10):989-993.

37. Takagi M, Hayashi H, Yoshida T: The effect of osmolarity on metabolism and morphology in adhesion and suspension chinese hamster ovary cells producing tissue plasminogen activator. Cytotechnology 2000 32(3):171-179.

38. Hieter P, Boguski M: Functional genomics: It's all how you read it. Science 1997, 278(5338):601-602.

39. Schena M, Heller RA, Theriault TP, Konrad K, Lachenmeier E, Davis RW: Microarrays: biotechnology's discovery platform for functional genomics. Trends Biotechnol 1998, 16(7):301-306.

40. Tao H, Bausch C, Richmond C, Blattner FR, Conway T: Functional genomics: Expression analysis of Escherichia coli growing on minimal and rich media. J Bacteriol 1999, 181(20):6425-6440.

41. Toscano WA, Oehlke KP: Systems biology: new approaches to old environmental health problems. Int J Environ Res Publ Health 2005 2(1):4-9.

42. Anthony JC: The promise of psychiatric enviromics. Brit J Psychiat 2001, 40:58-11. 
43. van Os J, Rutten BPF, Poulton R: Gene-Environment interactions in schizophrenia: Review of epidemiological findings and future directions. Schizophrenia Bull 2008, 34(6):1066-1082.

44. Geladi P, Kowalski BR: Partial Least-Squares regression - A tutorial. Anal Chim Acta 1986, 185:1-17.

45. Burger C, Carrondo MJT, Cruz H, Cuffe M, Dias E, Griffiths JB, Hayes K, Hauser H, Looby D, Mielke C, et al: An integrated strategy for the process development of a recombinant antibody-cytokines fusion protein expressed in BHK cells. Appl Microbiol Biot 1999, 52(3):345-353.

46. Teixeira A, Cunha AE, Clemente JJ, Moreira JL, Cruz HJ, Alves PM, Carrondo MJT, Oliveira R: Modelling and optimization of a recombinant BHK-21 cultivation process using hybrid grey-box systems. J Biotechnol 2005, 118(3):290-303.

47. Carinhas N, Bernal V, Monteiro F, Carrondo MJT, Oliveira R, Alves PM: Improving baculovirus production at high cell density through manipulation of energy metabolism. Metab Eng 2010, 12(1):39-52.

48. Stephanopoulos GN, Aristidou AA, Nielsen J: Metabolic engineering: Principles and methodologies. San Diego: Academic Press; 1998

49. Gagneur J, Klamt S: Computation of elementary modes: a unifying framework and the new binary approach. BMC Bioinformatics 2004, 5:1-21.

doi:10.1186/1752-0509-5-92

Cite this article as: Teixeira et al:: Cell functional enviromics: Unravelling the function of environmental factors. BMC Systems Biology 2011 5:92.

\section{Submit your next manuscript to BioMed Central and take full advantage of:}

- Convenient online submission

- Thorough peer review

- No space constraints or color figure charges

- Immediate publication on acceptance

- Inclusion in PubMed, CAS, Scopus and Google Scholar

- Research which is freely available for redistribution

Submit your manuscript at www.biomedcentral.com/submit
C Biomed Central 